\title{
Analysis of an independent tumor suppressor locus telomeric to Tp53 suggested Inpp5k and Myo1c as novel tumor suppressor gene candidates in this region
}

Carola Hedberg Oldfors ${ }^{1}$, Diego Garcia Dios ${ }^{1 \dagger}$, Anna Linder ${ }^{1 \dagger}$, Kittichate Visuttijai ${ }^{1,2}$, Emma Samuelson ${ }^{1}$, Sandra Karlsson ${ }^{2}$, Staffan Nilsson ${ }^{3}$ and Afrouz Behboudi $2^{* *}$

\begin{abstract}
Background: Several reports indicate a commonly deleted chromosomal region independent from, and distal to the TP53 locus in a variety of human tumors. In a previous study, we reported a similar finding in a rat tumor model for endometrial carcinoma (EC) and through developing a deletion map, narrowed the candidate region to $700 \mathrm{~kb}$, harboring 19 genes. In the present work real-time GPCR analysis, Western blot, semi-quantitative GPCR, sequencing, promoter methylation analysis, and epigenetic gene expression restoration analyses (5-aza-2'-deoxycytidine and/or trichostatin A treatments) were used to analyze the 19 genes located within the candidate region in a panel of experimental tumors compared to control samples.

Results: Real-time qPCR analysis suggested Hicl (hypermethylated in cancer 1), Inpp5k (inositol polyphosphate-5phosphatase K; a.k.a. Skip, skeletal muscle and kidney enriched inositol phosphatase) and Myolc (myosin 1c) as the best targets for the observed deletions. No mutation in coding sequences of these genes was detected, hence the observed low expression levels suggest a haploinsufficient mode of function for these potential tumor suppressor genes. Both Inpp5k and Myo1c were down regulated at mRNA and/or protein levels, which could be rescued in gene expression restoration assays. This could not be shown for Hic1.

Conclusion: Innp5K and Myolc were identified as the best targets for the deletions in the region. INPP5K and MYO1C are located adjacent to each other within the reported independent region of tumor suppressor activity located at chromosome arm 17p distal to TP53 in human tumors. There is no earlier report on the potential tumor suppressor activity of INPP5K and MYO1C, however, overlapping roles in phosphoinositide (PI) 3-kinase/Akt signaling, known to be vital for the cell growth and survival, are reported for both. Moreover, there are reports on tumor suppressor activity of other members of the gene families that INPP5K and MYO1C belong to. Functional significance of these two candidate tumor suppressor genes in cancerogenesis pathways remains to be investigated.
\end{abstract}

Keywords: Endometrial carcinoma, 17p13.3, RNO10q24-q25, Tp53, Hic1, Inpp5k, Skip, Myo1c

\footnotetext{
*Correspondence: afrouz.behboudi@his.se

${ }^{\dagger}$ Equal contributors

${ }^{2}$ Tumor Biology Research Group, School of Bioscience, University of Skövde,

SE-54128 Skövde, Sweden

Full list of author information is available at the end of the article
} 


\section{Background}

Allelic loss at $17 \mathrm{p}$ is one of the most frequently reported chromosomal alterations in a variety of human malignancies [1-4]. There are quite a few tumor suppressor loci reported in this region among which TP53 on $17 \mathrm{p} 13.1$ is the most prominent one reported to be altered in over $40 \%$ of all tumors [5]. However, several studies clearly provided evidence for presence of an independent, commonly deleted region or regions at $17 \mathrm{p} 13.3$, suggesting the existence of an additional tumor suppressor gene(s) distal to the TP53 locus [6-9]. Despite many efforts, no definite candidate(s) has yet been identified.

We have previously reported a similar observation of an independent tumor suppressor locus distal to $T p 53$ in an experimental model for endometrial carcinoma (EC) [10]. Cytogenetic and molecular analysis of ECs developed in a rat model for this malignancy revealed frequent allelic losses/deletions in the proximal to middle part of rat chromosome 10 (RNO10) [11-13]. Through deriving onco-tree models based on allelic imbalance (AI) data, we determined the likely order of allelic loss events along RNO10 as well as their relationship to each other [14]. In the analysis one of the small regions of recurrent allelic loss located at RNO10q24-q25 was placed closest to the root of the onco-tree models, suggesting this region to harbor early and important genetic alterations. The classical tumor suppressor gene $T p 53$ is located close to this chromosomal segment and thus was selected as the candidate. Subsequent analysis, however, revealed that Tp53 was not the only target [10], and in fact, the observed pattern for AI, chromosomal breaks and deletions suggested that major selection was directed against a region located close to, but distal of Tp53. This independent, commonly deleted chromosomal segment at RNO10q24-q25 is homologous to the frequently reported loci of tumor suppressor activity on $17 \mathrm{p} 13.3$ in several human malignancies [6-9]. Using the experimental tumor model, we developed a detailed deletion map and narrowed down the size of the region to a chromosomal segment of about $700 \mathrm{~kb}$ [10]. There are 19 genes located in this segment, including several putative tumor suppressor genes, notably Hic1 (hypermethyalted in cancer 1), Ovca1 (ovarian cancer-associated gene 1), and Ovca2 (ovarian cancer-associated gene 2) [9]. In the present work, we subjected the 19 genes located in this candidate region to expression analysis in a panel of rat EC and nonmalignant endometrium samples. Statistical analysis of qPCR results combined with subsequent gene mutation screening along with epigenetic and protein expression analyses suggested Inpp5k and Myolc as the most prominent target tumor suppressor candidates in this region.

\section{Results}

Real-time quantification PCR

To determine potential target genes for the observed frequent AI/deletions distal to the Tp53 gene [10], we determined the expression profile of all the 19 genes located in this region in a panel of 28 rat primary tumor and seven NME (non-malignant endometrium) cell cultures. Nine of the genes displayed significant decreased expression in EC compared to the NME samples (nominal $P$-value $<0.05$, Fig. 1, Table 1 ). The question was then whether the observed reduced expression of these nine genes was due to the physical deletion of the genetic material or other regulatory mechanisms. To address this, we used earlier $\mathrm{CGH}, \mathrm{AI}$, and FISH data $[12,13,15,16]$ and divided the 28 tumors analyzed in the gene expression assays into two groups: ECs with deletion/AI and those without deletion/AI spanning the $700 \mathrm{~kb}$ candidate chromosomal segment. We subsequently used this new grouping of tumors and reanalyzed the real-time RT-PCR results to determine whether there existed a correlation between physical deletion and the observed lowered expression of the nine genes among the tumor groups. Lowered expression of five genes (Hic1, Rpa1, Inpp5k, Myolc and Crk) was found to lack correlation with the physical deletion in the region (Table 1), suggesting the involvement of other regulatory mechanisms in silencing of these genes. Fold change in expression of Rpal was minimal and Crk is mostly known as an oncogene. The remainder three genes were thus selected as candidates for further analysis.

\section{Western blot analysis of Hic1, Inpp5k and Myo1c expressions}

Based on qPCR results, five rat ECs with differential expressions of Hic1, Inpp5k and Myo1c and from different genetic backgrounds as well as three NME samples, as control (Additional file 1: Table S1), were selected for Western blot analysis to validate the qPCR data. The analysis revealed no decrease in the expression of Hic1 protein in EC compared to the NME samples (Fig. 3a and $3 \mathrm{~b})$. The Myo1c protein expression levels correlated well with the observed qPCR fold changes and down regulation of Myo1c protein expression was detected in four out of five ECs compared to the NME samples (Fig. 3a and 3b). Our attempts for expression analysis of Inpp5k protein in this sample set failed, most likely due to the problem with specificity of the purchased antibody for rat samples that is produced against the human INPP5K protein.

\section{DNA sequencing and mutation analysis of Hic1, Inpp5k and Myo1c}

To screen Hic1, Inpp5k and Myolc genes for potential inactivating mutations, the entire coding regions of 
Table 1 Changes in relative expression of 19 genes within tumor suppressor region located at 62.3 - 63.0 Mb (RNO10q24) distal to Tp53

\begin{tabular}{|c|c|c|c|c|c|c|}
\hline \multirow[t]{2}{*}{ Transcript } & \multirow{2}{*}{$\begin{array}{l}\text { Position } \\
\text { (Mb) }\end{array}$} & \multicolumn{3}{|c|}{ Group EC vs NME } & \multicolumn{2}{|c|}{ Group EC with vs EC without loss at RNO10q24 } \\
\hline & & P-value & $<0.05$ & Fold change & P-value & $<0.05$ \\
\hline Est1a & 62.23 & 0.002 & $x$ & -2.3 & 0.03 & $x$ \\
\hline Hic1 & 62.47 & 0.0004 & $x$ & -4.9 & 0.2 & \\
\hline Ovca2 & 62.48 & 0.760 & & & & \\
\hline Dph1 & 62.49 & 0.973 & & & & \\
\hline Rtn4rl1 & 62.50 & 0.008 & $x$ & -4.5 & 0.02 & $x$ \\
\hline Rpa1 & 62.61 & 0.003 & $x$ & -1.9 & 1.1 & \\
\hline Smyd4 & 62.66 & 0.918 & & & & \\
\hline Serpinf1 & 62.71 & 0.293 & & & & \\
\hline Serpinf2 & 62.75 & 0.324 & & & & \\
\hline Wdr81 & 62.76 & 0.00 .5 & $x$ & -1.7 & 0.01 & $x$ \\
\hline TIcd2 & 62.79 & 0.218 & & & & \\
\hline Prf8 & 62.81 & 0.054 & & & & \\
\hline Rilp & 62.83 & 0.108 & & & & \\
\hline Scarf1 & 62.84 & 0.811 & & & & \\
\hline Slc43a2 & 62.85 & 0.247 & & & & \\
\hline Pitpn & 62.91 & 0.0003 & $x$ & -2.2 & 0.0004 & $x$ \\
\hline Inpp5k & 62.95 & 0.0003 & $x$ & -6.8 & 0.2 & \\
\hline Myolc & 62.99 & 0.000003 & $x$ & -4.8 & 0.1 & \\
\hline Crk & 63.02 & 0.00001 & $x$ & -3.2 & 0.4 & \\
\hline
\end{tabular}

Nine genes displayed significant decreased expression (nominal p-value $<0.05$ ) in EC compared to the NME samples. Tumors were then divided into two groups of with and without deletion/Al at RNO10q24 and the gene expression data for the nine down regulated genes were reanalyzed. Down regulation of five genes (marked in bold) was not correlated with physical deletion at RNO10q24

Fold changes and P-values of the different transcripts. The negative values of fold change represent a decreased expression. FC shown only for genes displaying $P$-values $<0.05$ between NME vs EC

Equal variances assumed. t-test for equality of means

Hic1, Inpp5k and Myo1c, as well as the promoter region of Myolc were sequenced using genomic DNA from tumor cell cultures and the parental rat strains (BDII, $\mathrm{BN}$ and SPRD) as template (Additional file 1: Table S1). No mutation in Hic1, Inpp5k or Myo1c was found.

Sequence analysis of Hicl and Myolc revealed 10 strain specific variations within these genes: five in Hicl (two in noncoding and three in coding sequences, Additional file 2: Table S5) and five in Myo1c (all in noncoding sequences, Additional file 2: Table S5). It is important to note that one of the identified SNPs in the coding sequence of Hic1 gene (at nt 62474413) results in a non-conservative amino acid substitution in BDII strain, from nonpolar alanine (Ala177) to polar threonine (Thr177, Additional file 2: Table S5).

\section{Methylation analysis of Hic1, Inpp5k and Myo1c}

To investigate the potential involvement of epigenetic inactivation of the three candidate genes in tumors without physical deletion, a panel of tumors with or without deletion in the region, and with differential expressions of the Hic1, Inpp5k and Myo1c, were subjected to DNA methylation analysis. The overall methylation profile of CpG islands in Hic1, Inpp5k and Myolc promoter regions was characterized using the bisulfite genomic sequencing method in a panel of 18 samples, including 14 ECs (Additional file 1: Table S1), three parental strains (BDII, BN and SPRD) and a positive control sample (methylase treated BDII). This analysis was complemented with the MSP method for parts of Hicl promoter region for which bisulfite sequencing was not successful (data not shown). Using the CpG Island Searcher program (http://www.uscnorris.com/cpgislands2/cpg.aspx), the promoter region of the Hicl gene was found at position -1352 to -476 (877 bp, including 47 CpG sites). Bisulfite genomic sequencing (for $26 \mathrm{CpGs)}$ combined with MSP analysis (for the remainder $21 \mathrm{CpGs)} \mathrm{revealed}$ partial promoter methylation in 9 out of $14(64 \%)$ of the tumors (Fig. 3c). The Inpp5k promoter was located at position -517 to +53 ( $570 \mathrm{bp}$, containing $53 \mathrm{CpG}$ sites). The location of the promoter region of the Myolc gene was found at position -2000 to -1111 (890 bp, including $55 \mathrm{CpG}$ sites). No methylation was detected in the Inpp5k and Myolc promoters in the tumors analyzed, 
whereas different degrees of promoter methylation were detected in the Hicl. The detected Hicl promoter methylation was not correlating with the Hic1 expression at RNA and/or protein levels Fig. 3c.

\section{Restoration of gene expression after 5-Aza-dC and/or TSA treatments}

To investigate whether other epigenetic mechanisms might be involved in the reduction of expression of the three candidate genes we subjected a selected panel of cells to restoration of gene expression treatments. We selected four EC cell lines with reduced Myo1c, Hic1, and/or Inpp5k expression (NUT12 and NUT50 with, and NUT51 and NUT98 without deletion/AI in the candidate region RNO10q24-q25) as well as REF (rat embryonic fibroblast) cells for the analysis. Gene expression analysis at both RNA and protein levels, as expected, revealed no restoration of Hic1, Innp5k and Myo1c expressions after 5-Aza-dC and/or TSA treatments in the control cell REF (Fig. 4). The analysis also showed no or a marginal restoration (only after AZA treatment in NUT51) of gene expression for Hic1 after either or both treatments in the tumor samples tested Fig. 4a, d and e). RT-PCR analysis revealed a strong restoration of Inpp5k expression following 5-Aza-dC and/or TSA treatments in three of the tumors analyzed (NUT50, NUT51 and NUT98, Fig. 4b). For the Myolc gene, a marginal restoration of gene expression after the treatments was detected in RT-PCR analysis, in particular for NUT50 (Fig. 4c). Analysis of Myolc protein, however, was more conclusive and clearly showed that expression of Myolc protein was partially restored after the treatments, especially in NUT12 (with physical deletion/AI in the region) and NUT98 (without physical deletion/AI in the region) as well as in NUT50 (with deletion/AI in the region) after the combined treatments (Fig. 4e).

\section{Discussion}

In the present work and by using a set of wellcharacterized experimental EC tumor samples, and through a candidate gene approach, we characterized the frequently reported independent region of tumor suppressor activity in human tumors located telomeric to TP53. We subjected all 19 genes located in this chromosomal segment to gene expression analysis in a panel of 28 rat EC and seven control non-malignant endometrium (NME) samples. Nine genes showed significant reduced expression in EC compared to NME samples (Fig. 1, Table 1). Interestingly, two of the known tumor suppressor genes located in this region, Ovca1 (ovarian cancer-associated gene 1) and Ovca2, were not among these.

The question was then whether the observed reduced expression of these nine genes was due to the physical deletion of the genetic material or other regulatory mechanisms. To address this, we used earlier CGH, AI, and FISH data $[12,13,15,16]$ and divided the 28 tumors analyzed in the gene expression assays into two groups: ECs with deletion/AI and those without deletion/AI spanning the $700 \mathrm{~kb}$ candidate chromosomal segment. We subsequently used this new grouping of tumors and re-analyzed the real-time RT-PCR results to determine whether there existed a correlation between physical deletion and the observed lowered expression of the nine genes between the tumor groups (Fig. 2, Table 1). Our interest was to identify gene(s) showing no such correlation, indicating that the observed reduction in expression of these gene(s) was not only due to the physical deletion of the genetic material. The results revealed that lowered expression levels of five genes, Hic1, Rpa1, Inpp5k, Myo1c and Crk were independent of the observed physical deletions (Fig. 2, Table 1). Among these, Hic1, Inpp5k and Myo1c showed strong and highly significant down regulation in EC compared to the control samples (Table 1) and thus were selected as the best candidates. Rpal and Crk were not appealing as candidates: Rpal showed only a rather moderate down regulation in gene expression analysis (Table 1 ) and $\mathrm{Crk}$ (v-crk sarcoma virus CT10 oncogene homolog, avian) is mainly recognized as an oncogene in cancer studies [17-19].

In qPCR experiments, we found that Hicl, Innp5k and Myo1c were always expressed in the tumors, although at very low levels. According to Knudson's theory of inactivation of tumor suppressor genes [20], if Hic1, Inpp5k and Myolc are to behave as classical tumor suppressor genes, it is expected that both alleles of the genes be inactivated in the tumor material. We, therefore, hypothesized that the remaining allele of these genes might have become inactivated through mutation/s. However, in gene sequencing experiments, no mutation was found in coding sequences of these three genes, suggesting a potential haploinsufficient mode of function for these candidate tumor suppressor genes. In gene sequencings we identified ten SNPs in intronic, noncoding and/or coding sequences of the Hicl and Myolc genes (Additional file 2: Table S5). Identification of these SNPs offered the possibility to confirm our earlier AI/deletion data $[11,13]$ and also to make a detailed deletion map with information on the homozygote/hemizygote pattern of the observed deletions spanning the candidate region in the tumors.

Our interest was then to determine whether gene products of these three candidate genes were down regulated in the tumors. To this end, we examined expression of Hic1, Innp5k and Myo1c proteins in a selected panel of five ECs compared to three NME samples using Western blot. The result for Myo1c showed a good 


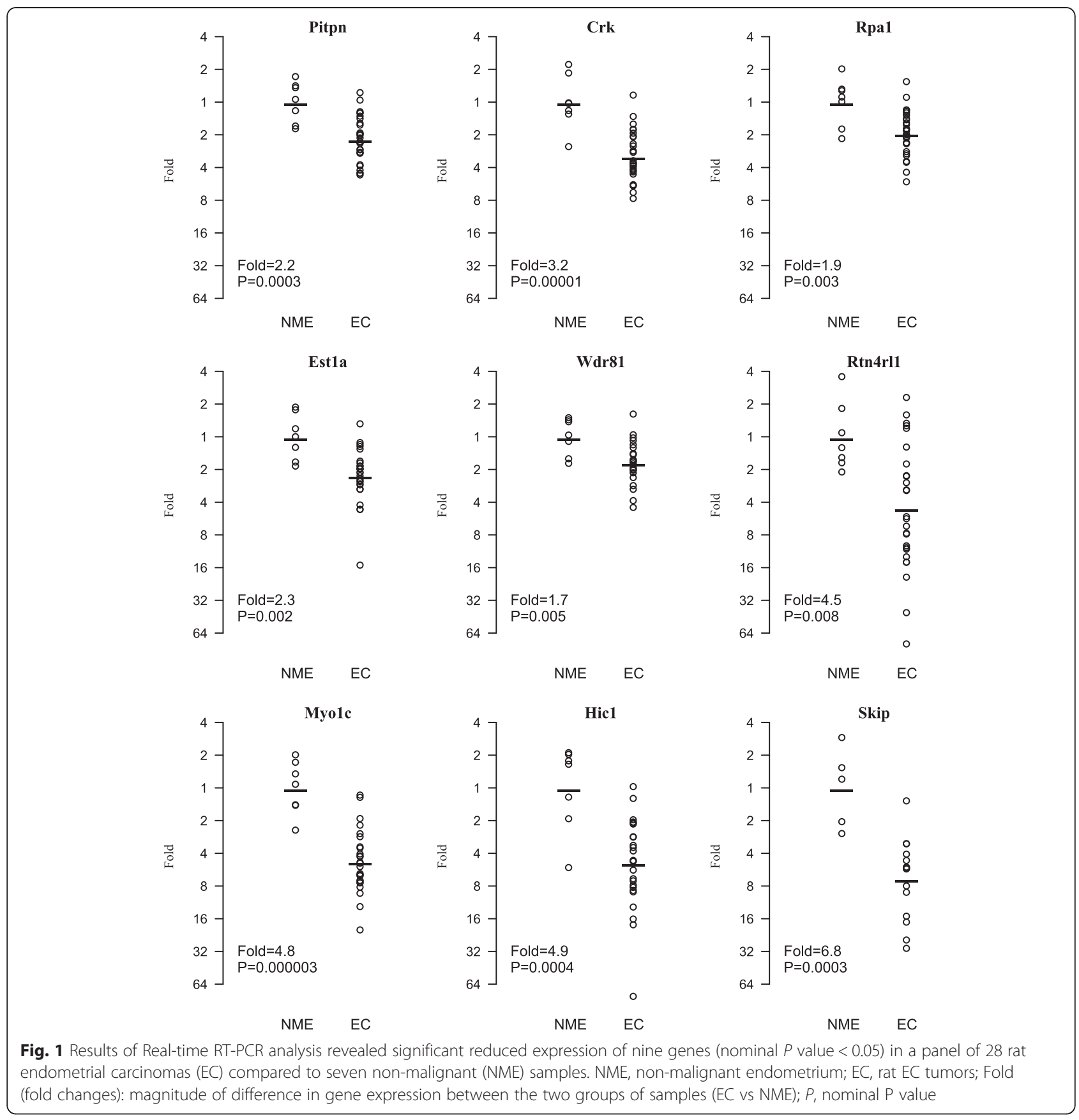

correlation between the $\mathrm{qPCR}$ results and protein expression level in the tumor samples, indicating that Myo1c protein was in fact down regulated in the majority of EC tumors tested compared to the control samples (Fig. 3a and b). However, such a correlation was not detected for Hic1 as it was shown that expression of Hic1 protein was not down regulated in the tumors, even in those showing a rather strong Hic1 down regulation at their mRNA level (NUT12, NUT51 and NUT98; Fig. 3a and b). No result was obtained for the Inpp5k protein, most likely due to the lack of specificity of the available human antibody for the rat samples.

There were a number of tumors that did not harbor $\mathrm{AI} /$ deletion in the candidate region, but nevertheless displayed a significant down-regulation of Hic1, Inpp5k and/or Myo1c (Table 1). The question was then whether other regulatory machineries, namely epigenetic regulation, were involved. To address this, we screened promoter regions of Hicl, Inpp $5 k$ and Myolc for methylation at $\mathrm{CpG}$ islands. No methylation was detected in the Inpp5k and Myolc promoter regions in any of the 


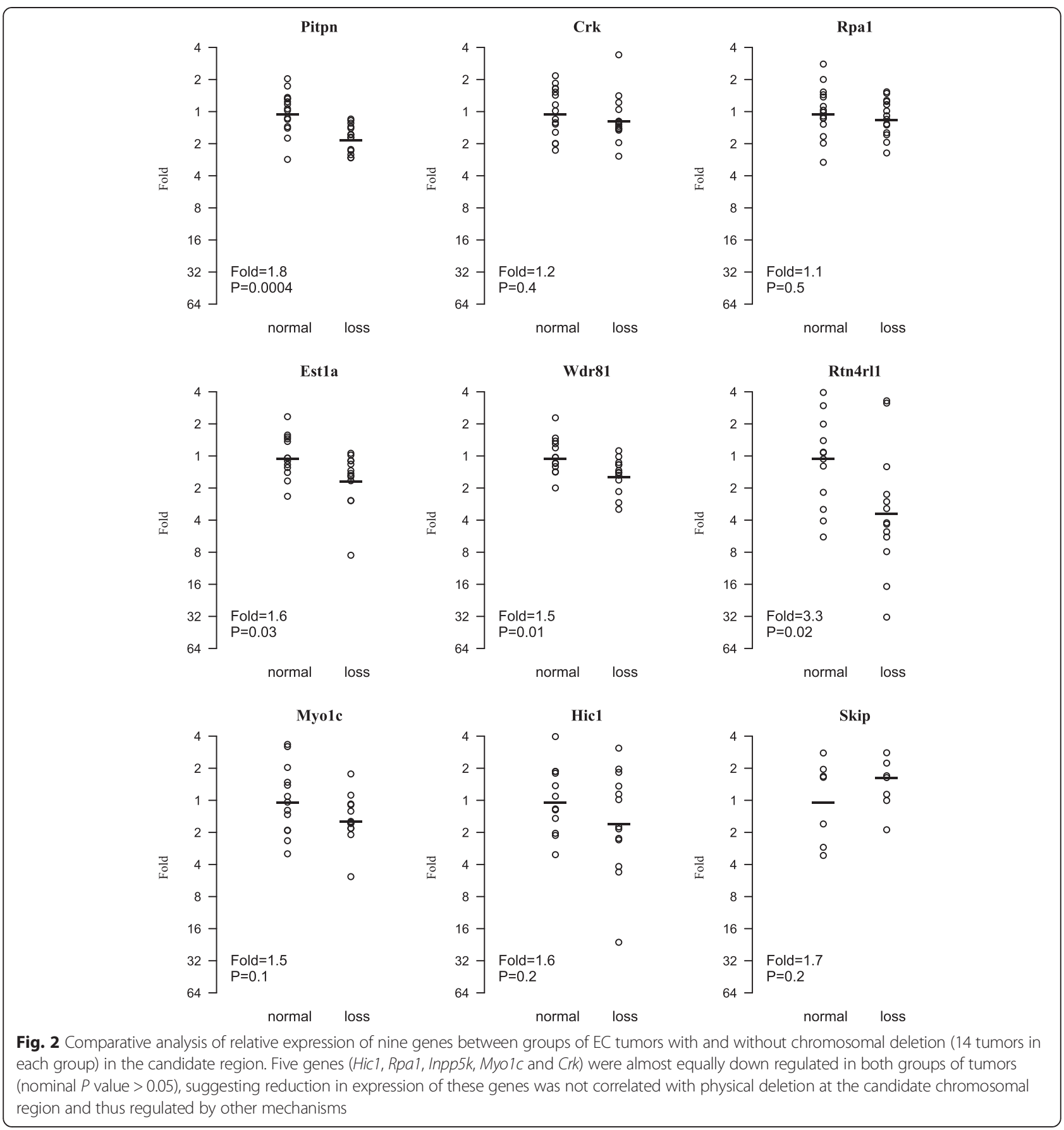

tumors tested, whereas partial DNA methylation was detected in Hic1 promoter in $64 \%$ of tumors tested (Fig. 3c). No correlation, however, between the observed methylation status of Hicl promoter and the level of Hic1 transcript was detected in tumor samples (Fig. 3c).

It is quite common that genes with critical functions are under restricting regulation by several promoters. Moreover, histone deacethyaltion is an alternative epigenetic regulation mechanism that may also result in the gene silencing. To examine whether other potential promoter(s) might have been involved in the expression regulation of Hic1, Inpp5k and Myolc as well as to study the potential involvement of histone deacethylation in expression regulation of these genes, we treated cell lines with demethylating agent 5-Aza-dC and/or deacetylating inhibitor TSA. Surprisingly, Hic1 expression was not specifically restored after the treatments in the samples, especially not in those that showed partial methylation in the Hic1 promoter region (NUT12 and NUT51, Figs. 3c, 4a, d and e). This result suggested that the 

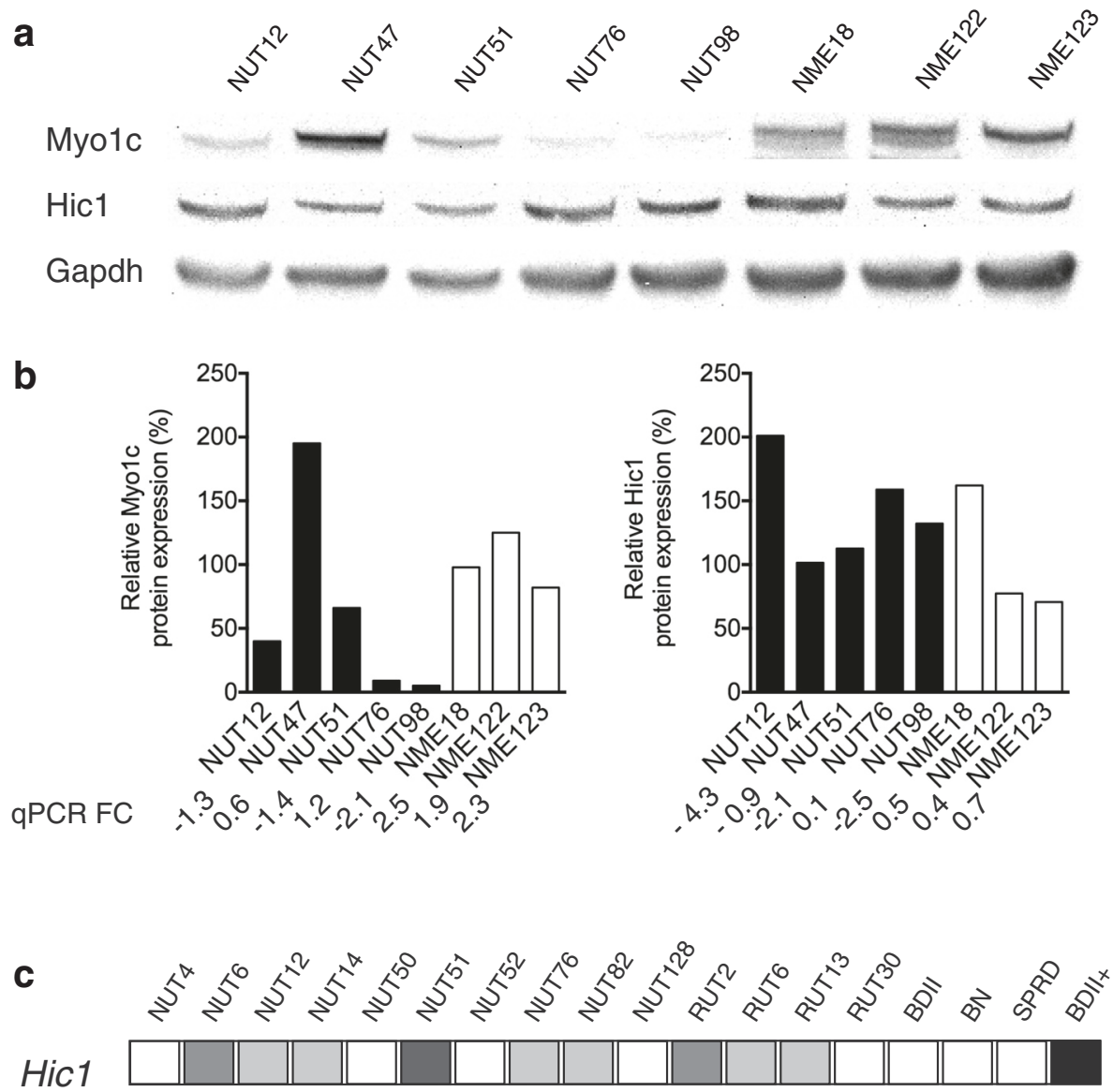

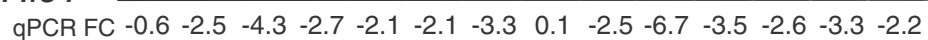
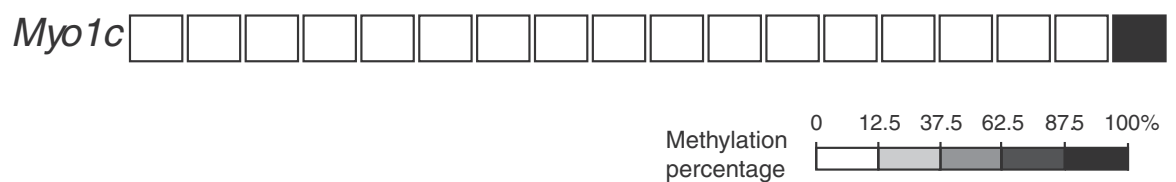

Fig. 3 a and $\mathbf{b}$ Analysis of Myo1c and Hic1 protein expression in five EC and three NME samples. The results shown are representative of three independent experiments. Myo1c protein displayed down regulated in four out of five ECs tested, whereas Hic1 protein was not down regulated in the EC samples, even in those that in GPCR experiment showed a strong lowered expression of Myo1c transcript. The analysis additionally showed a very good correlation between expression of Myo1c at RNA and protein levels in the tumors tested, whereas such correlation was not detected for Hic1. EC: enodemetrail carcinoma; NME: non-malignant endometrium. c Methylation status of CpG islands in promoter regions of Hic1 (26 CpG sites) and Myolc in a panel of 14 ECs, three parental strains (BDII, BN and SPRD) and one positive control (BDII +) samples. Methylation frequencies are color coded (black, ranges of gray and white) in five white/gray/black grades corresponding to percentages of CpG sites that were found methylated in each sample. For comparison, qPCR results for the Hicl gene is presented as fold changes (FC) for each tumor, where negative and positive fold change values represent reduced and increased expressions, respectively. As shown, a correlation between methylation score at the Hic1 promoter and expression of this gene in tumors is lacking. No methylation was identified in the promoter region of Myolc

observed methylation pattern in the Hic1 promoter region in EC tumors, rather than being an epigenetic silencing mechanism, is likely to be a general structural feature for this gene. In agreement with this, dense hypermethylation of one of the HIC1 alleles has earlier been reported in a number of normal human tissues, including kidney [21], and histologically normal and benign hyperplastic prostate tissues [22].
Restoration of gene expression was detected for Myo1c and Inpp5k genes in the tumor samples at RNA and/or protein levels (Fig. 4b-e). Notably, the observed gene expression restoration for Myolc protein was irrespective of $\mathrm{AI} /$ deletion in the candidate region, as NUT12 with AI/deletion and NUT98 without AI/deletion showed strong restoration of Myolc expression after either or both treatments (Fig. 4e). These data 
a
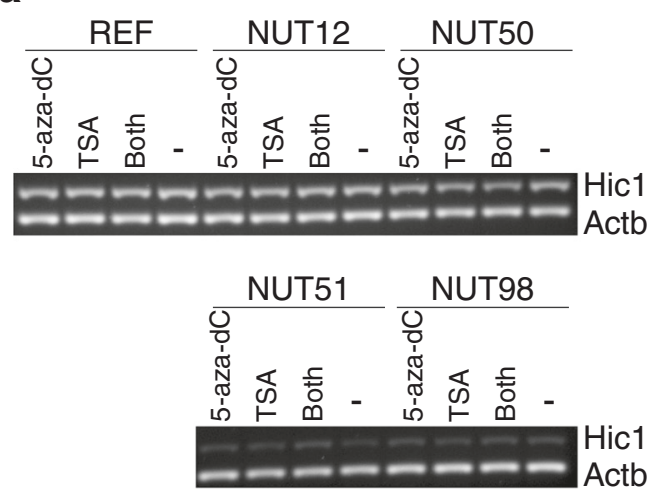

C

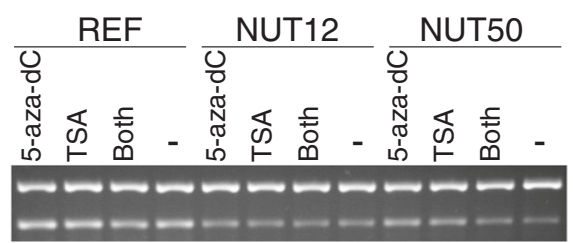

Actb

Myo1c
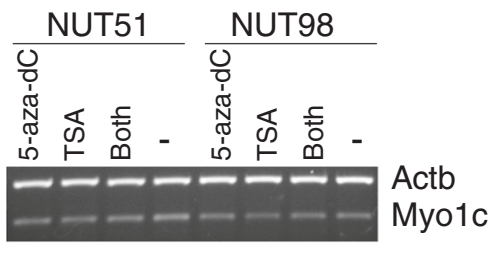

b
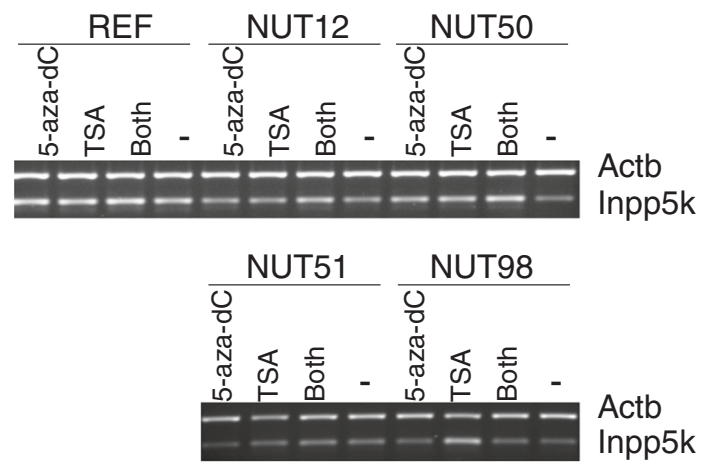

d

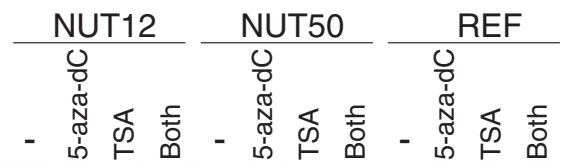

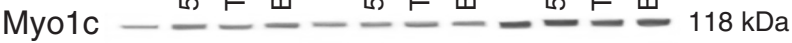

Hic1 - - _ - - - $-100 \mathrm{kDa}$

Gapdh - - - - - - - - $37 \mathrm{kDa}$

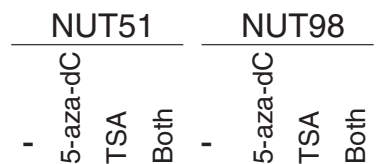

Myo1c - ஸ્

Hic1 1 -ニニニニニ $118 \mathrm{kDa}$

Gapdh - - - - - $37 \mathrm{kDa}$

e
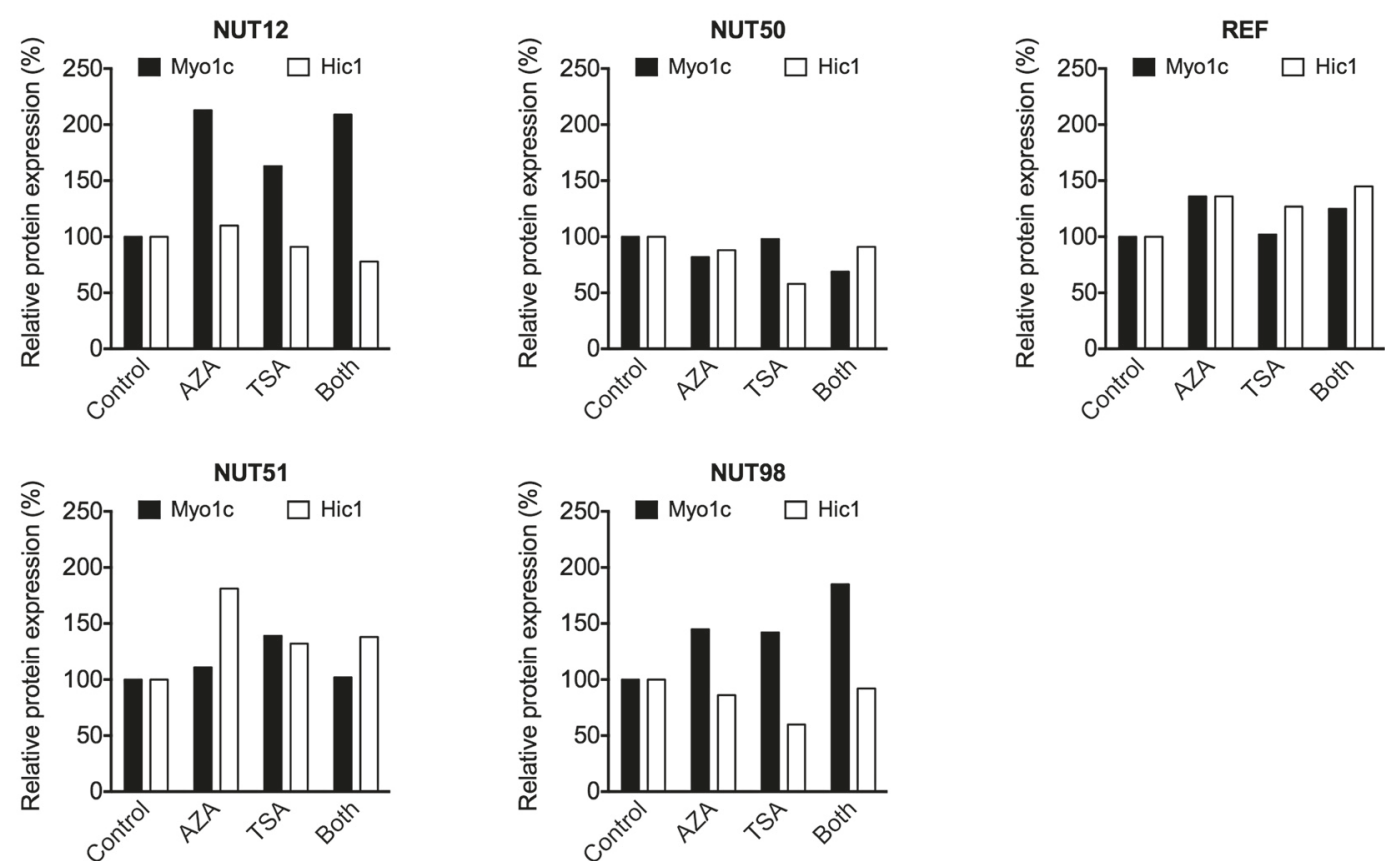

Fig. 4 (See legend on next page.) 
(See figure on previous page.)

Fig. 4 Expression patterns of Hic1, Inpp5k and Myo1c after treatments with 5-Aza-dC and/or TSA in four tumors (NUT12 \& NUT50 with and NUT51 \& NUT98 without deletion in the candidate region) and one control (rat embryonic fibroblast, REF) samples. The results shown are representative of two independent experiments. a Hic1 mRNA expression, b Inpp5k mRNA expression, c Myo1c mRNA expression, d protein expression of Hicl and Myolc, and e quantification of Western blot analysis for Hic1 and Myolc expressions. As shown, expression of Hic1 was not specifically restored in these assays (only after AZA treatment in one sample, NUT51), whereas expressions of Myo1c (at both mRNA and protein levels) and Inpp5k (at mRNA level) were restored after either or both of these treatments. Interestingly, restoration of Myo1c gene expression was observed in both tumors with (NUT12) and without (NUT98) Al/deletion in the region

suggest that epigenetic gene silencing might have an important role in inactivation of Myolc and Inpp5k tumor suppressor candidates.

To summarize, qPCR analysis of 19 genes located within the commonly deleted region distal to $T p 53$ in experimental ECs, suggested Hic1, Inpp5k and Myo1c as the best candidate tumor suppressor genes in this region. No mutation was detected in the coding sequences of the retained alleles, suggesting a potential haploinsufficient mode of function for these candidate tumor suppressor genes. Hic1, Inpp5k, and Myolc showed reduced expression in tumor samples irrespective of the presence or absence of physical deletion in the candidate region, suggesting the potential involvement of alternative gene silencing regulatory mechanism(s). We found a rather normal expression of Hic1 protein in the tumors, even in those that showed down regulation of Hic1 at the mRNA levels, indicating lack of correlation between expression of Hic1 at mRNA and protein levels. Promoter methylation analysis revealed partial methylation of Hic1 promoter in a number of tumors. Nevertheless, there was again no correlation between the presence or absence of Hicl promoter methylations and gene expression levels in the tumors. Moreover, Hic1 expression could not specifically be restored after treatments with demethylating and histone deacetylase inhibitor agents. In contrast, while promoter methylation was not detected in Inpp5k and Myo1c promoters, down regulation of Inpp5k and Myo1c were detected in EC tumors, and this could be rescued after the 5-Aza-dC and TSA treatments, even in tumors without $\mathrm{AI} /$ deletion in the candidate tumor suppressor region. Taken together, results from the present work exclude Hic1 as a fitting candidate and provide evidence for Inpp $5 k$ and Myolc as two attractive candidates for the observed independent tumor suppressor activity at the neighborhood of $T p 53$.

INPP5K (inositol polyphosphate-5-phosphatase $\mathrm{K}$, also known as SKIP, skeletal muscle and kidney enriced inositol phosphatase) is a member of the inositol polyphosphate 5-phosphatases family [23] with a poorly characterized function in vivo. The inositol polyphosphate 5-phosphatases family is known as negative regulators of PI 3-kinase signaling [24]. Analysis of the role of INPP5K in insulin-stimulated cells indicated that endogenous INPP5K might be one of the key regulators of insulin signaling in skeletal muscle and adipocytes for glucose homeostasis [25]. INPP5K was identified as a 5'inositol phosphatase that hydrolyzes phosphatidylinositol 3,4,5-triphosphate (PI-3,4,5- $\mathrm{P}_{3}$ ) and phosphatidylinositol 4,5-bisphosphate (PI-4,5- $\left.\mathrm{P}_{2}\right)$ to negatively regulate intracellular phosphatidylinositol 3-kinase signaling. It is thus suggested that INPP5K exerts its functions through direct binding to $\mathrm{PIP}_{3}$ or forming a complex with molecules located downstream of PI 3-kinase. Activated PI 3-kinase generates $\mathrm{PIP}_{3}$ that in turn activates the downstream target $\mathrm{AKT}$, which then positively regulates a range of cellular functions, including actin rearrangement, protein synthesis, cell metabolism, cell cycle (G1-S transition) and cell survival [24]. So far, there is no report on potential involvement of INPP5K in cancer progression. However, another member of the phosphoinositide phosphatase family, PTEN, has already been identified as a haploinsufficient tumor suppressor gene [26] and its inactivation has been implicated in a variety of human cancers, including endometrial carcinoma. Therefore, it is tempting to speculate that INPP5K might likewise be potentially involved in carcinogenesis.

The gene adjacent to Inpp5k, and the second candidate tumor suppressor gene identified in the present work, is the molecular motor myosin 1c (Myo1c). Myo1c exerts overlapping functions in phosphoinositide (PI) 3kinase/AKT (PI3K/AKT) signaling as those of Inpp5k. Myosin $1 \mathrm{C}$ is a widely expressed vertebrate unconventional myosin-I isoform that concentrates in perinuclear regions, on ruffling cell membranes, and within stereocilia of hair cells. Increasing evidence points to the role of myosin 1c in many signaling cascades, from the integrin-dependent signaling involved in cell migration to the signaling events underlying insulin resistance (reviewed in [27]). Myo1c is a lipid raft-associated motor protein that is specifically involved in the recycling of lipid raft membrane and proteins that regulate plasma membrane plasticity, cell motility and pathogen entry $[28,29]$. MYO1C binds tightly and specifically to $\mathrm{PIP}_{2}$ [30], an important second messenger involved in a variety of crucial cellular functions, including regulation of the actin cytoskeleton and signal transduction in insulin and AKT signaling pathways. This protein is additionally involved in glucose uptake in muscle and adipocytes through controlling of movement of intracellular GLUT4- 
containing vesicles to the plasma membrane [31]. It has been shown that insulin-dependent phosphorylation of Myo1c is required for GLUT4 translocation and transport of glucose through phosphoinositide (PI) 3-kinase/AKT pathway [32, 33]. There is no earlier report on potential tumor suppressor activity of this gene, but several other members of the myosin-I gene family have been reported as cancer-related genes, including tumor suppressor gene $M Y O 18 B$ in lung, ovarian and colorectal cancer [34] and involvement of $M Y O 1 F$ in chromosomal translocation and gene fusion in infant acute monocytic leukemia [35].

\section{Conclusions}

In conclusion, our data suggested Inpp $5 k$ and Myolc as potential candidates located adjacent to each other within the reported independent tumor suppressor loci distal to $T p 53$. There is no earlier report on their potential involvement in carcinogenesis, but earlier studies have clearly suggested a regulatory role for these genes in PI3K/Akt pathway, which is known to be vital to the growth and survival of cancer cells [36]. Moreover, other members of gene families that INPP5K and MYO1C belong to are suggested to function as tumor suppressor genes in a variety of cancer types [26,34]. Details of potential functional contributions of these two genes to cancerogenesis remain to be further investigated.

\section{Methods}

\section{Animal crosses and experimental tumor material}

All animal experiments were approved by the local ethical committee (Institute of Laboratory Animal Science and Central Animal Facility, Hannover Medical School). Animals of the inbred BDII rat strain are genetically predisposed to spontaneous EC, with an incidence of more than $90 \%$ in virgin females before the age of 24 months [37, 38]. EC tumors developed in F1, F2 and backcross (N1) progeny of crosses between BDII females and males from two EC non-susceptible strains, $\mathrm{BN} / \mathrm{Han}$ and SPRD-Cu3/Han [39, 40], were included in the present study (Additional file 1: Table S1). In some cases no malignant cells were detected in the removed cell mass from animals when pathologically characterized. Chromosomal analysis of these samples revealed only minor numerical chromosomal changes (unpublished data). In this study these tissues represent normal or pre-malignant endometrium and thus are referred to as non-malignant endometrium (NME, Additional file 1: Table S1). At necropsy, tumor specimens were collected from animals for DNA extraction using a standard phenol-based method in the Genepure ${ }^{\text {ra }} 341$ Nucleic Acid Purification System (PE Applied Biosystems) [12]. Small pieces of fresh tumor as well as NME tissues were used to set up primary cell cultures. From the cell cultures, DNA and total RNA were extracted using the GenElute kit (Mammalian Total RNA Kit, Sigma).

\section{Real-time quantitative PCR}

Expression of 19 genes (Table 2) was analyzed in a panel of $28 \mathrm{EC}$ and seven NME samples using Real-time quantitative PCR as described previously [41]. Quantification and normalization of the results was performed by the standard-curve method. Briefly, a standard curve was prepared in each PCR assay for all genes using serial dilutions $(1: 1,1: 3,1: 9,1: 18,1: 36$, and 1:72) of one of the tumor samples (RUT30) and/or a commercially available rat RNA mix (Stratagene, La Jolla, CA, USA). The mean $\mathrm{C}_{\mathrm{T}}$-value for triplicates was calculated, $\mathrm{C}_{\mathrm{T}}$ values of the serial dilutions were used to interpolate standard curves for each gene and data therein was used to determine concentration or copy number of each gene in every test sample. To normalize the results, three housekeeping genes (Gapdh, $\beta$-actin and Rps9) were included in the analysis, among which Rps9 (ribosomal protein S9) showed the lowest variations in $\Delta C_{\mathrm{T}}$ levels regardless of the cell type [41] and thus was selected and used as the internal reference for normalization of expression of all 19 genes in the samples. Logarithmic expression levels were then compared with Welch's t-test for adjusted expression levels based on standard curves as well as expression of Rps 9 in EC tumors compared to NME samples.

\section{Western blot}

A panel of five EC and three NME samples was analyzed by western blot to examine levels of Hic1, Inpp5k and Myo1c protein expression using 1:500 rabbit anti-Hic1 (H8539, Sigma Aldrich), 1:1000 rabbit anti-Inpp5k (S8948, Sigma Aldrich), 1:600 rabbit anti-Myo1c (HPA001768, Sigma Aldrich), and 1:500 dilution anti-Gapdh (sc-825778, Santa Cruz, Biotechnology) according to the standard protocol (Additional file 1: Table S1, Fig. 3a and b).

\section{DNA sequencing of Hic1, Inpp5k and Myo1c}

A panel of 32 EC tumors was selected for mutation sequencing of Hic1 and Myolc (Additional file 1: Table S1). The Inpp5k gene was sequenced in 18 tumors. Primer pairs were designed using the Primer3 program and synthesized by a commercial supplier (SIGMAGenosystem, Cambridge, UK). PCR primers set corresponding to the coding sequences of Hicl, Inpp $5 k$ and Myolc genes were amplified and screened for mutations (Additional file 3: Table S2). For the Myo1c gene the promoter region was also sequenced.

PCR amplification products were purified using GFX PCR DNA and gel Band Purification Kit (Amersham Pharmcia Biotech, Piscataway, NJ). Using ABI PRISM ${ }^{\circ}$ BigDye $^{\bullet}$ Terminator v1.1 or 3.1 Cycle Sequencing Kit 
Table 2 Genes located within tumor suppressor region between 62.3-63.0 Mb on RNO10 at band 10q24 which is homologous to human chromosome band $17 p 13.3$

\begin{tabular}{|c|c|c|c|c|}
\hline Start (Mb) & Gene & Description & Accession no. & Assay ID \\
\hline 62.23 & Est1a & Telomerase-binding protein EST1A (Ever shorter telomeres $1 \mathrm{~A}$ ) & NM_001105808 & Rn01466713_m1 \\
\hline 62.47 & Hicl & Hypermethylated in cancer 1 & NM_001107021 & CM-Hic1 \\
\hline 62.48 & Orca2 & Candidate tumor suppressor OVCA2 & NM_001109036 & CM-Ovca2 \\
\hline 62.49 & Ovcal & Candidate tumor suppressor OVCA1, Dph2l1 & NM_001105809 & CM-Ovca1 \\
\hline 62.50 & Rtn4rl1 & Reticulon 4 receptor-like 1 & NM_181377 & Rn01648154_m1 \\
\hline 62.61 & Rpal & Replication protein A1 & NM_001047843 & Rn01460703_g1 \\
\hline 62.66 & Smyd4 & SET and MYND domain containing 4 & NM_001105810 & CM-Smyd4 \\
\hline 62.71 & Serpinf1 & Serine (or cysteine) peptidase inhibitor, clade F, member 1 & NM_177927 & Rn00709999_m1 \\
\hline 62.75 & Serpinf2 & Serine (or cysteine) peptidase inhibitor, clade F, member 2 & NM_001011892 & Rn01464596_m1 \\
\hline 62.76 & Wdr81 & Wdr81, WD repeat domain 81 & NM_001134360 & Rn01460094_m1 \\
\hline 62.79 & Tlcd2 & TLC domain containing 2 & XM_573142.2 & Rn01465130_m1 \\
\hline 62.81 & Prpf8 & Pre-mRNA processing factor 8 & XM_001080695 & Rn01472027_g1 \\
\hline 62.83 & Rilp & Rab interacting lysosomal protein & NM_001105811 & Rn01465376_g1 \\
\hline 62.84 & Scarf1 & Scavenger receptor class $F$, member 1 & NM_001107022 & CM-Scarf1 \\
\hline 62.85 & $5 / c 43 a 2$ & Solute carrier family 43, member 2 & NM_001105812 & CM-SIc43a2 \\
\hline 62.91 & Pitpna & Phosphatidylinositol transfer protein, alpha & NM_017231 & Rn01464411_m1 \\
\hline 62.95 & Skip & Skeletal muscle and kidney enriched inositol phosphatase & NM_001013859 & Rn01466844_g1 \\
\hline 62.99 & Myolc & Myosin IC & NM_023092 & Rn00576538_m1 \\
\hline 63.02 & Crk & V-crk sarcoma virus CT10 oncogene homolog (avian) & NM_019302 & Rn00467066_m1 \\
\hline $1 q 12$ & Rps9 & Ribosomal protein $\$ 9$ & NM_031108.2 & Rn01530912_g1 \\
\hline $4 q 42$ & Gapdh & Glyceraldehyde-3-phosphate dehydrogenase & NM_017008.3 & Rn99999916_s1 \\
\hline $12 \mathrm{p} 11$ & $\beta$-actin & Beta-actin & NM_031144 & Rn00667869_m1 \\
\hline
\end{tabular}

TaqMan assays used in quantitative real-time PCR for the genes in the region as well as three housekeeping genes are presented

CM: Custom made

(Applied Biosystems), the purified DNA fragment were subjected to sequencing according to the protocol provided by the manufacturer. Sequencing products were separated on a denaturing polyacrylamide gel on a 3130xl Genetic Analyzer (Applied Biosystems) and analyzed using the software's Sequencing Analysis v5.2 and SeqScape v2.5 (Applied Biosystems).

\section{DNA methylation analysis}

A panel of 14 tumors was selected for promoter methylation analysis of Hic1, Inpp5k and Myolc (Additional file 1: Table S1). One $\mu \mathrm{g}$ DNA from 14 tumors as well as the three controls (parental strains: BDII, BN and SPRD) were denatured, treated by sodium bisulfite and purified using Epitect Bisulfite Kit according to manufacturer protocol (Qiagen). A positive control sample was produced through methylase treatment of DNA from BDII. This sample was then sodium bisulfite treated and processed at similar conditions as the test samples. We used web server CpG Island Searcher (URL: http:// www.uscnorris.com/cpgislands2/cpg.aspx) to predict location of $\mathrm{CpG}$ islands for both genes. Primers suitable for bisulphite sequencing were designed using the Meth-
Primer software (URL: http://urogene.org/methprimer/) and BiSearch web server (URL: http://bisearch.enzim.hu/) and synthesized by a commercial supplier (SIGMAGenosystem, Cambridge, UK). Promoter regions were PCR amplified using two different overlapping sets of primer for Hicl and three sets of primers for Myolc using treated DNA as template (Additional file 4: Table S3). In some cases it was necessary to perform semi-nested PCR to produce enough PCR products for sequencings. The methylation status was then analyzed using bisulphite sequencing as described earlier [42].

Our attempts to PCR amplify and sequence the middle part of the Hic1 promoter (-1082 to -781$)$ failed. This was mainly due to high density of CpGs in the sequence. We thus analyzed methylation status of this part of the Hic1 promoter using the MSP (methylation specific PCR) technique. Primer pair sequences and PCR conditions are available upon request

\section{5-aza-2'-deoxycytidine (5-Aza-dC) and/or trichostatin A (TSA) treatment}

Four EC cell lines (NUT12 and NUT50 with and NUT51 and NUT98 without deletion/AI at RNO10q24) 
with low expression of Hic1, Inpp5k and/or Myolc, and rat embryo fibroblasts (REF), were selected for the analysis. All cell cultures were treated with the demethylating agent 5-aza-2'-deoxycytidine (5-Aza-dC, SigmaAldrich Co, St Louis, MO, USA) and/or with the histone deacetylase inhibitor trichostatin A (TSA; Sigma-Aldrich). Concentrations of the agents for treatments were chosen based on an earlier study (Karlsson et al., unpublished data). Each cell line was seeded at low density in four separate flasks, treated with 5-Aza-dC, TSA, both 5-Aza-dC and TSA, or no treatment. For 5-Aza-dC treatments, cells were grown in medium containing $2.5 \mu \mathrm{M} 5$-Aza-dC for 96 hours, with medium and drug being refreshed twice after $48 \mathrm{~h}$ and $72 \mathrm{~h}$. For simultaneous 5-Aza-dC and TSA treatments, the procedure was as 5 -Aza-dC treatment, with the addition of $300 \mathrm{nM}$ TSA to the culture during the last 16 hours. For the TSA treatment as well as the negative control, medium was refreshed after $48 \mathrm{~h}$ and $72 \mathrm{~h}$, with the exception that for the TSA treatment, $300 \mathrm{nM}$ TSA was added to the culture during the last 16 hours. Two independent experiments for all treatments and tumor samples were performed. After 96 hours, total RNA was isolated from all samples using RNeasy Protect Mini kit (Qiagen) according to the manufacturer's protocol. Protein extraction was performed according to standard protocols.

\section{Semi quantitative RT-PCR}

Reverse-transcription polymerase chain reaction (RT-PCR) was performed on $1 \mu \mathrm{g}$ of total RNA using QuantiTect ${ }^{\circ}$ Reverse Transcription Kit (Qiagen) according to manufacturer's protocol. Semi quantitative RT-PCR was performed for Hic1, Inpp5k and Myo1c using $\beta$-actin as internal control in multiplex PCR. Primer pairs were designed using the Primer3 program and were synthesized by a commercial supplier (SIGMA-Genosystem, Cambridge, UK) (Additional file 5: Table S4). PCR amplification was performed for 26 cycles.

\section{Additional files}

Additional file 1: Table S1. Panel of cell cultures used in the present study. Different sets of samples were used for different analysis as: Mut, mutation sequencing (32 EC); qPCR (28 EC and seven NME), Real-time PCR; WB, Western blot (five EC and three NME); PM, promoter methylation (14 EC); AT, 5-Aza-dC/TSA treatments (four EC and one NME).

Additional file 2: Table S5. SNPs identified in the gene sequencing analysis based on the build RGSC3.4 data.

Additional file 3: Table S2. Primers used for sequencing the genes Hicl, Myolc and Inpp5k.

Additional file 4: Table S3. Primers used for bisulphite sequencing of the promotors of Hicl and Myo1C. For Hicl the predicted CpG island was $877 \mathrm{bp}$ and includes $47 \mathrm{CpG}$ sites. For Myo1c the predicted CpG island of $890 \mathrm{bp}$ and includes $55 \mathrm{CpG}$ sites.
Additional file 5: Table S4. Primers used for semi quantitative multiplex RT-PCR for Hic1, Inpp5k and Myo1c amplified in a multiplex PCR with $\beta$-actin as the internal control.

\section{Abbreviations}

Crk: v-crk sarcoma virus CT10 oncogene homolog, avian; EC: endometrial carcinoma; Hic1: hypermethylated in cancer 1); Inpp5k: inositol polyphosphate-5phosphatase K (also known as Skip); Ovca1: ovarian cancer-associated gene 1; Ovca2: ovarian cancer-associated gene 2; Myo1c: myosin 1c; NME: non-malignant endometrium; Rpa1: Replication protein A1; Skip: skeletal muscle and kidney enriched inositol phosphatase.

\section{Competing interests}

The authors declare that they have no competing interests.

\section{Authors' contributions}

CHO participated in the design of the study, carried out (together with FA) qPCR, gene expression restoration, gene sequencing and methylation experiments and drafted the manuscript. DGD and AL carried out gene mutation sequencing, methylation experiments, analyzed the produced data and participated in drafting of the corresponding parts of the manuscript. KV prepared material for WB analysis, participated in data analysis and preparation of figures. ES prepared the cell material and participated in the sequence alignment. SK participated in preparation of biological material, and data analysis. SN supervised and performed data analysis. $A B$ conceived the study, designed and coordinated the experiments, and drafted and finalized the manuscript. All authors read and approved the final manuscript.

\section{Acknowledgement}

We are grateful to Frida Abel, Stina Petersson, Emman Shubbar for technical assistance. The real-time PCR was performed at the Genomics Core Facility platform at the Sahlgrenska Academy, University of Gothenburg, which is funded by a grant from the Knut and Alice Wallenberg Foundation.

\section{Funding}

This work was supported by: Royal Swedish Academy of Sciences [grant number FOA07L575-A and FOA08H-185], Royal Physiographic Society in Lund (Nilsson-Ehle Foundation) [grant number 24900 and 24538], Swedish Society of Medicine [grant number 2009-22226], Gunvor and Ivan Svensson Foundation, Wilhelm and Martina Lundgren Foundation [grant number 339 and 324], Åke-Wieberg Foundation [grant number 946217602], Assar Gabrielsson Research Foundation for Clinical Cancer Research [grant number FB 08-11 and FB 08-23], Sahlgrenska University Hospital Foundation [grant number ALFGBG-24451], The Knowledge Foundation [HÖG12, 20120311].

\section{Author details}

'Department of Medical and Clinical Genetics, Sahlgrenska Academy, University of Gothenburg, SE-40530 Gothenburg, Sweden. ${ }^{2}$ Tumor Biology Research Group, School of Bioscience, University of Skövde, SE-54128 Skövde, Sweden. ${ }^{3}$ Institute of Mathematical Statistics, Chalmers University of Technology, SE-41296 Gothenburg, Sweden.

Received: 12 March 2015 Accepted: 23 June 2015

Published online: 14 July 2015

\section{References}

1. Beckmann MW, Niederacher D, Schnurch HG, Gusterson BA, Bender HG. Multistep carcinogenesis of breast cancer and tumour heterogeneity. Journal of Molecular Medicine-Jmm. 1997;75:429-39.

2. Bova GS, Isaacs WB. Review of allelic loss and gain in prostate cancer. World Journal of Urology. 1996;14:338-46.

3. Kaneko Y, Homma C, Maseki N, Sakurai M, Hata J. Correlation of Chromosome-Abnormalities with Histological and Clinical-Features in Wilms and Other Childhood Renal Tumors. Cancer Research. 1991;51:5937-42.

4. Sterkers Y, Preudhomme C, Lai JL, Demory JL, Caulier MT, Wattel E, et al, Acute myeloid leukemia and myelodysplastic syndromes following essential thrombocythemia treated with hydroxyurea: High proportion of cases with 17p deletion. Blood. 1998;91:616-22. 
5. Whibley C, Pharoah PDP, Hollstein M. p53 polymorphisms: cancer implications. Nature Reviews Cancer. 2009;9:95-107.

6. Guan XY, Sham JS, Tai LS, Fang Y, Li H, Liang Q. Evidence for another tumor suppressor gene at 17p13.3 distal to TP53 in hepatocellular carcinoma. Cancer genetics and cytogenetics. 2003;140:45-8.

7. Konishi H, Sugiyama M, Mizuno K, Saito H, Yatabe Y, Takahashi T, et al. Detailed characterization of a homozygously deleted region corresponding to a candidate tumor suppressor locus at distal 17p13.3 in human lung cancer. Oncogene. 2003;22:1892-905.

8. Roncuzzi L, Brognara I, Baiocchi D, Amadori D, Gasperi-Campani A. Loss of heterozygosity at 17p13.3-ter, distal to TP53, correlates with negative hormonal phenotype in sporadic breast cancer. Oncology reports. 2005;14:471-4.

9. Sarkar C, Chattopadhyay P, Ralte AM, Mahapatra AK, Sinha S. Loss of heterozygosity of a locus in the chromosomal region 17p13.3 is associated with increased cell proliferation in astrocytic tumors. Cancer Genetics and Cytogenetics. 2003;144:156-64.

10. Nordlander C, Karlsson S, Karlsson A, Sjoling A, Winnes M, Klinga-Levan K, et al. Analysis of chromosome 10 aberrations in rat endometrial cancer-evidence for a tumor suppressor locus distal to Tp53. Int J Cancer. 2007;120:1472-81.

11. Behboudi A, Levan G, Hedrich HJ, Klinga-Levan K. High-density marker loss of heterozygosity analysis of rat chromosome 10 in endometria adenocarcinoma. Genes, Chromosomes and Cancer. 2001;32:330-41.

12. Helou K, Walentinsson A, Beckmann B, Johansson A, Hedrich HJ, Szpirer C, et al. Analysis of genetic changes in rat endometrial carcinomas by means of comparative genomic hybridization. Cancer Genet Cytogenet. 2001;127:118-27.

13. Nordlander C, Behboudi A, Levan G, Levan KK. Allelic imbalance on chromosome 10 in rat endometrial adenocarcinomas. Cancer Genet Cytogenet. 2005;156:158-66.

14. Chen L, Nordlander C, Behboudi A, Olsson B, Levan KK. Deriving evolutionary tree models of the oncogenesis of endometrial adenocarcinoma. Int J Cancer 2007:120:292-6

15. Behboudi A, Hedrich HJ, Szpirer C, Levan G, Klinga-Levan K. Loss of heterozygosity analysis of rat chromsome 10 in endometrial adenocarcinoma. Rat Genome. 2000;6:77.

16. Hamta A, Adamovic T, Helou K, Levan G. Cytogenetic aberrations in spontaneous endometrial adenocarcinomas in the BDII rat model as revealed by chromosome banding and comparative genome hybridization. Cancer Genetics and Cytogenetics. 2005;159:123-8.

17. Kobashigawa Y, Sakai M, Naito M, Yokochi M, Kumeta H, Makino Y, et al. Structural basis for the transforming activity of human cancer-related signaling adaptor protein CRK. Nature Structural \& Molecular Biology. 2007;14:503-10.

18. Nishihara H, Tanaka S, Tsuda M, Oikawa S, Maeda M, Shimizu M, et al. Molecular and immunohistochemical analysis of signaling adaptor protein Crk in human cancers. Cancer Letters. 2002;180:55-61.

19. Wang L, Tabu K, Kimura T, Tsuda M, Linghu H, Tanino M, et al. Signaling adaptor protein Crk is indispensable for malignant feature of glioblastoma cell line KMG4. Biochemical and Biophysical Research Communications. 2007;362:976-81.

20. Knudson AG. A two-mutation model for human cancer. Advances in Viral Oncology. 1987;7:1-7.

21. Makos M, Nelkin BD, Reiter RE, Gnarra JR, Brooks J, Isaacs W, et al. Regional DNA Hypermethylation at D17s5 Precedes 17p Structural-Changes in the Progression of Renal Tumors. Cancer Research. 1993;53:2719-22.

22. Morton RA, Watkins JJ, Bova GS, Wales MM, Baylin SB, Isaacs WB. Hypermethylation of chromosome 17P locus D17S5 in human prostate tissue. Journal of Urology. 1996;156:512-6.

23. Astle MV, Seaton G, Davies EM, Fedele CG, Rahman P, Arsala L, et al. Regulation of phosphoinositide signaling by the inositol polyphosphate 5-phosphatases. lubmb Life. 2006;58:451-6.

24. Engelman JA, Luo J, Cantley LC. The evolution of phosphatidylinositol 3-kinases as regulators of growth and metabolism. Nature Reviews Genetics. 2006;7:606-19.

25. Ijuin T, Takenawa T. SKIP negatively regulates insulin-induced GLUT4 translocation and membrane ruffle formation. Molecular and Cellular Biology. 2003;23:1209-20.

26. Maehama T, Dixon JE. The tumor suppressor, PTEN/MMAC1, dephosphorylates the lipid second messenger, phosphatidylinositol 3,4,5-trisphosphate. Journal of Biological Chemistry. 1998;273:13375-8.
27. Bond LM, Brandstaetter H, Kendrick-Jones J, Buss F. Functional roles for myosin 1C in cellular signaling pathways. Cell Signal. 2013;25:229-35.

28. Brandstaetter $\mathrm{H}$, Kendrick-Jones J, Buss F. Myo1 $\mathrm{c}$ regulates lipid raft recycling to control cell spreading, migration and Salmonella invasion. J Cell Sci. 2012;125:1991-2003

29. Oh H, Kim H, Shin B, Lee KH, Yeo MG, Song WK. Interaction of crk with Myosin-1C participates in fibronectin-induced cell spreading. Int J Biol Sci. 2013;9:778-91.

30. Hokanson DE, Ostap EM. Myo1c binds tightly and specifically to phosphatidylinositol 4,5-bisphosphate and inositol 1,4,5-trisphosphate. Proc Natl Acad Sci U S A. 2006;103:3118-23.

31. Bose A, Guilherme A, Robida SI, Nicoloro SM, Zhou QL, Jiang ZY, et al. Glucose transporter recycling in response to insulin is facilitated by myosin Myo1c. Nature. 2002;420:821-4

32. Holman GD, Sakamoto K. Regulating the Motor for GLUT4 Vesicle Traffic. Cell Metabolism. 2008:8:344-6.

33. Yip MF, Ramm G, Larance M, Hoehn KL, Wagner MC, Guilhaus M, et al. CaMKII-Mediated Phosphorylation of the Myosin Motor Myolc Is Required for Insulin-Stimulated GLUT4 Translocation in Adipocytes. Cell Metabolism. 2008;8:384-98.

34. Nishioka M, Kohno T, Tani M, Yanaihara N, Tomizawa Y, Otsuka A, et al MYO18B, a candidate tumor suppressor gene at chromosome 22q12.1, deleted, mutated, and methylated in human lung cancer. Proc Natl Acad Sci U S A. 2002;99:12269-74.

35. Taki T, Akiyama M, Saito S, Ono R, Taniwaki M, Kato Y, et al. The MYO1F, unconventional myosin type $1 \mathrm{~F}$, gene is fused to MLL in infant acute monocytic leukemia with a complex translocation involving chromosomes 7, 11, 19 and 22. Oncogene. 2005:24:5191-7.

36. Engelman JA. Targeting PI3K signalling in cancer: opportunities, challenges and limitations. Nature reviews Cancer. 2009;9:550-62.

37. Deerberg F, Kaspareit J. Endometrial carcinoma in BDII/Han rats: model of a spontaneous hormone-dependent tumor. Journal of the National Cancer Institute. 1987:78:1245-51.

38. Kaspareit-Rittinghausen J, Deerberg F, Rapp K. Mortality and incidence of spontaneous neoplasms in BDII/Han rats. Zeitschrift für Versuchstierkunde. 1987;30:209-16.

39. Roshani L, Mallon P, Sjostrand E, Wedekind D, Szpirer J, Szpirer C, et al. Genetic analysis of susceptibility to endometrial adenocarcinoma in the BDII rat model. Cancer Genetics and Cytogenetics. 2005:158:137-41.

40. Roshani L, Wedekind D, Szpirer J, Taib Z, Szpirer C, Beckman B, et al. Genetic identification of multiple susceptibility genes involved in the development of endometrial carcinoma in a rat model. International Journal of Cancer. 2001;94:795-9.

41. Samuelson E, Hedberg C, Nilsson S, Behboudi A. Molecular classification of spontaneous endometrial adenocarcinomas in BDII rats. Endocr Relat Cancer. 2009;16:99-111.

42. Caren H, Fransson S, Ejeskar K, Kogner P, Martinsson T. Genetic and epigenetic changes in the common Ip36 deletion in neuroblastoma tumours. British Journal of Cancer. 2007;97:1416-24.

\section{Submit your next manuscript to BioMed Central and take full advantage of:}

- Convenient online submission

- Thorough peer review

- No space constraints or color figure charges

- Immediate publication on acceptance

- Inclusion in PubMed, CAS, Scopus and Google Scholar

- Research which is freely available for redistribution 